\title{
Students step up
}

\author{
Universities and institutions in the United States are getting ready to celebrate nanotechnology and its \\ achievements for National Nanotechnology Day. Lisa Friedersdorf, Quinn Spadola and Brendan Ryan \\ share students' plans for the occasion.
}

$\mathrm{S}$ uperhydrophobic coatings that allow you to rinse off your phone under running water; carbon nanotube yarns that generate electricity; nano-enabled filters that provide clean water; nanomedicine that may lead to a universal flu vaccine: National Nanotechnology Day (\#NationalNanoDay) is an opportunity to raise awareness of these and other applications of nanotechnology. This annual celebration on and around October the 9 th (for $10^{-9}$, of course!) is a broad collection of events and activities in the US national nanotechnology community. Last year, professional societies dedicated special webpages and blog posts to nanotechnology, and published white papers and articles about the current state of nanoscience. Universities held nanoscale image exhibits and competitions, and conducted outreach events in their local communities. Experts and visionaries participated in 'Nano Nuggets', a series of videos on the impact of nanotechnology. And classrooms across the country took on the challenge to run the 100-billion-nanometre dash (\#100BillionNanometers, \#100BNM).

This year, National Nanotechnology Day will be celebrated with podcasts; superhero contests; image, video and entrepreneurship competitions; seminars and workshops; and many other activities. Students from the Nano and Emerging Technologies Student Network (NextTech) are planning to participate, too. NextTech is made up of clubs run by undergraduate students at universities and colleges across the United States. Throughout the year, NextTech chapters organize a wide variety of events, such as research mixers that bring together eager students and faculties with research opportunities in their labs; seminar series with academic and industry leaders speaking on a host of topics; and laboratory tours at universities and local industrial or federal facilities. Some of the groups coordinate research projects, and others focus on entrepreneurship and encourage student start-ups. Many of the groups also engage in outreach in their communities to excite schoolchildren about science and engineering. For National Nanotechnology

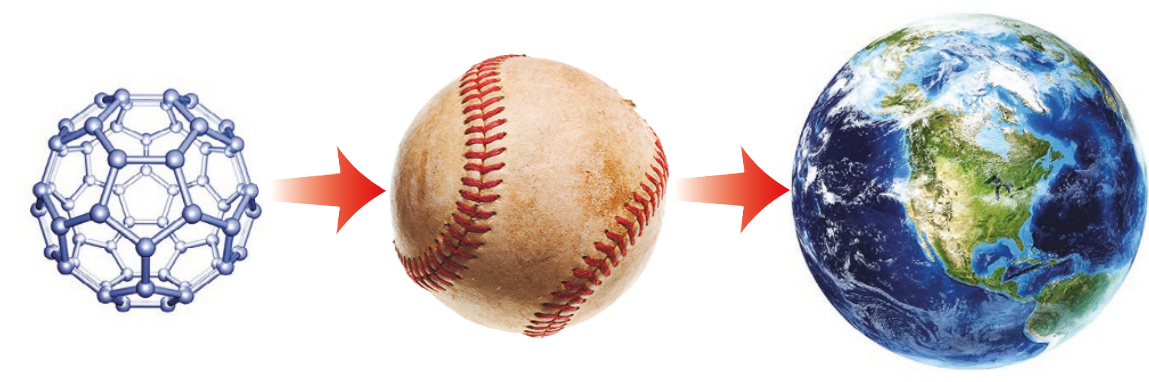

Day, the students are organizing several activities, a few of which are listed below.

At the University of Washington, students are hosting an 'Introduce a Girl to Nanotechnology' fair. Modelled after a successful event focused on photonics aimed at encouraging girls' interest in STEM, the fair will consist of activities about nanotechnology including resources to help students build a graphene circuit or a solar cell. The fair will also feature information highlighting 'Nano Ladies' - female researchers working in nanotechnology.

Virginia Tech's Collegiate Nanotechnology Society (CNS) is promoting the NanoWorld Challenge for 9 October 2017. The challenge is inspired by the popular factoid used to explain the nanoscale: that the size of the 'buckyball' fullerene relative to a softball is about the same as a softball relative to the Earth. For this social media challenge, people are asked to make their own softballsized globe and tweet an image of it using the \#NationalNanoDay or \#NanoWorld tag. Additionally, CNS will host a screening of a movie about nanotechnology.

Students from the Society for Emerging and NanoTechnologies (SENT) at the University of Central Florida will present their research at the NanoFlorida 2017, an annual gathering of faculty and student researchers along with industry representatives from across the state.

The Georgetown University Technology and Engineering Club (GUTEC) will be conducting tours of the Georgetown Nanoscience and Microtechnology Laboratory, a user facility featuring stateof-the-art fabrication and characterization equipment. The new Nano and Emerging Technologies (NET) club at George Mason University is working with two international semiconductor companies to schedule tours of local nanoelectronics fabrication facilities. Nanotechnology fellows from George Washington University may also join these tours.

The Nano and Emerging Technologies (NExT) club at the University of Virginia and students at St John's University in New York are planning to host and promote lectures focused on the intersection of nanotechnology with energy sciences and chemistry, respectively.

The Material Advantage chapter at the University of Puerto Rico at Mayagüez is supporting a half-day nano activity that will include demonstrations on nanotechnology and a movie screening. And many students, including members of GUTEC, CNS, NET and students from St John's University, plan to promote and run the 100-billionnanometre dash.

These are just a few examples of activities planned to raise awareness and celebrate nanotechnology. What will you do for National Nanotechnology Day? We'd love to hear from you.

LISA E. FRIEDERSDORF and QUINN A. SPADOLA are at the National Nanotechnology Coordination Office, Alexandria, Virginia 22314, USA. BRENDAN RYAN is at the Collegiate Nanoscience Society at Virginia Tech University, Blacksburg, Virginia 24061, USA. e-mail: Ifriedersdorf@nnco.nano.gov; qspadola@nnco.nano.gov; brendanr@vt.edu 